\title{
Marfan Syndrome Type II
}

National Cancer Institute

\section{Source}

National Cancer Institute. Marfan Syndrome Type II. NCI Thesaurus. Code C75007.

Marfan syndrome caused by mutations in the TGFBR2 gene. 